(D) Check for updates

Cite this: Analyst, 2020, 145, 4782

Received 27th March 2020, Accepted 12th June 2020

DOI: 10.1039/d0an00602e

rsc.li/analyst

\section{Quantitative UHPSFC-MS analysis of elemental sulfur in mineral oil via derivatisation with triphenylphosphine: application to corrosive sulfur-related power transformer failure}

\author{
Sérgio B. Garcia, ${ }^{a}$ Julie Herniman, ${ }^{a}$ Peter Birkin, (D) a James Pilgrim, ${ }^{b}$ Paul Lewin, ${ }^{\text {b }}$ \\ Gordon Wilson, ${ }^{c}$ G. John Langley ${ }^{\star a}$ and Richard C. D. Brown (D) *a
}

\begin{abstract}
An ultrahigh-performance supercritical fluid chromatographymass spectrometry (UHPSFC-MS) method has been developed as a rapid and reliable analytical method for the detection and quantification of elemental sulfur in mineral transformer oil. The method described in this paper is based on the selective reaction of elemental sulfur with triphenylphosphine (TPP). The derivatisation of elemental sulfur requires minimal sample preparation and resulted in the formation of a single compound, namely triphenylphosphine sulfide (TPPS). This derivative is quantified from the complex oil composition using electrospray ionisation-mass spectrometry (ESI-MS) in selected ion monitoring (SIM) mode, and the reported UHPSFC-MS method allows detection and quantification of the derivative at ppb levels. As sulfur contamination in mineral transformer oil has been linked to costly failures of oil/paper-based power transformers due to corrosion, the analytical approach is demonstrated through its application to mineral oil samples from in service and decommisioned power transformers. The method is ideal as a routine test or to confirm the presence of elemental sulfur in samples where corrosion has occurred.
\end{abstract}

\section{Introduction}

Oil insulation systems are used by numerous power apparatus. Key components in power networks, like power transformers, use mineral oil not only for cooling but also for electrical insulation. High voltage (HV), super grid transformers cost millions of pounds. Therefore, reliability and economic viability of power distribution is highly dependent on availability, performance and longevity of power transformers. ${ }^{1}$

\footnotetext{
${ }^{a}$ School of Chemistry, University of Southampton, Southampton SO17 1BJ, UK. E-mail: rcb1@soton.ac.uk, G.J.Langley@soton.ac.uk; Tel: +44 (0)2380594108

${ }^{b}$ The Tony Davies High Voltage Laboratory, University of Southampton, Southampton SO17 1BJ, UK

${ }^{c}$ National Grid, National Grid House, Warwick Technology Park, Gallows Hill, Warwick CV34 6DA, UK
}

In the last decades, the risk of failure in electrical equipment owing to the presence of corrosive sulfur in the mineral oil has increased. This risk has been understood for many decades but actual failures world-wide were very rare until around 2000. The International Council on Large Electric Systems (CIGRÉ) estimated the total number of failures due to corrosive sulfur to be in the region of 100 between 2000 and 2009. ${ }^{2}$ A number of the cases where associated with the presence of silver sulfide in silver-coated contacts, mainly in the tap-changers. ${ }^{3}$ Formation of surface metal sulfide will lead to overheating of the contacts followed by the detachment of conductive particles. These particles can cause breakdown and rated voltage stress. ${ }^{2}$ Transformer failures and/or repairs are expensive and should be avoided whenever possible. Outages if not predicted in advance can be expensive, not only for generators but also for networks. To avoid unexpected failures, diagnostic measures are necessary. Standard methods for corrosive sulfur detection in mineral oil have been published. ${ }^{4-8}$ These methods are not specific for $\mathrm{S}_{8}$, they are time consuming and subjective due to a visual rating process. Hence, analytical methods that allow rapid, reliable and reproducible quantification of $\mathrm{S}_{8}$ in mineral transformer oil are needed.

Several analytical techniques have been applied to determine $\mathrm{S}_{8}$ in hydrocarbons, but none specifically in mineral insulating oil. The use of gas chromatography (GC) and a number of variants (e.g., use of a selective detector for sulfur) can be found in the literature. ${ }^{9-13}$ Other methods include the use of polarography, ${ }^{14,15}$ liquid chromatography (LC), ${ }^{16}$ and GC-Inductively Coupled Plasma-MS (GC-ICP-MS) ${ }^{17,18}$ There are several issues that can arise when applying these methods to quantify $S_{8}$ in transformer mineral oil. In mineral oil, at room temperature elemental sulfur exists in an allotropic equilibrium $\left(\ldots S_{6} \rightleftharpoons S_{7} \rightleftharpoons S_{8} \ldots\right)$ with $S_{8}$ being the predominant allotrope, ${ }^{19}$ at elevated temperatures, as present in a standard GC injector, the allotropic equilibrium shifts to lower mass allotropes and this ensures that elemental sulfur does not elute from GC columns as a single and narrow chromatographic peak. Mineral oil has miscibility issues with reversed phase 


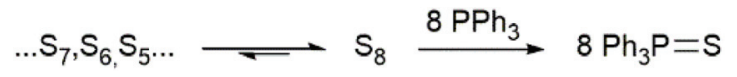

Fig. 1 Derivatisation reaction.

HPLC solvents, while normal phase HPLC is more challenging to interface to MS. Even coupled with GC, ICP-MS systems would suffer from matrix interference due to the myriad hydrocarbons present in mineral oil. ${ }^{20}$ UHPSFC can overcome the solubility issues related to the mineral oil, and when combined with a suitable derivatisation method and ESI-MS detection, may deliver simplified detection with minimal interference from the matrix.

One derivatisation method, consists of the reaction of sulfur, independent of its allotropic composition, to give a single stable compound, namely triphenylphosphine sulfide (TPPS, Fig. 1). ${ }^{21}$ This derivatisation method has been employed to determine elemental sulfur in different media. ${ }^{16,22,23}$ Another clear advantage of the derivatisation is an almost 9-fold increase in the mass concentration of the sulfur containing species $\left(1 \mu \mathrm{g} \mathrm{mL} L^{-1}\right.$ of sulfur $\rightarrow 9.18 \mu \mathrm{g} \mathrm{mL}^{-1}$ of TPPS). ${ }^{21}$

Herein, we describe a novel UHPSFC-MS method via derivatisation with TPP that allows the detection of elemental sulfur in mineral oil down to ppb levels. The reported method is capable of maintaining a high sensitivity and accuracy when applied to real mineral transformer samples.

\section{Materials}

\section{Chemicals}

Toluene (High-performance liquid chromatography (HPLC) grade) and methanol (Liquid Chromatography-Mass Spectrometry (LC-MS) grade) were purchased from Fisher Scientific (Loughborough, UK). Food grade carbon dioxide was purchased from BOC Special Gases. Elemental sulfur (99.998\%), TPP (99\%), TPPS (98\%) and ammonium acetate were purchased from Sigma-Aldrich (Gillingham, UK). Formic acid was purchased from Riedel-de Haën (Seelze, Germany) and National Grid plc (London, UK) kindly supplied representative mineral oil samples.

\section{Mineral oil samples}

National Grid plc provided mineral oil samples for analysis, some of which are believed to possess corrosive properties and all with varying compositions and chemistries. The samples were collected from a diverse range of transformers, presenting different working conditions and ages.

\section{Experimental}

\section{Analytical procedure}

Stock solution and calibration. A working stock solution of TPPS was made in 50:50 mineral oil/toluene (v/v) at a concen- tration of $100 \mu \mathrm{g} \mathrm{mL}^{-1}$. Dilutions of the working solution with 50:50 mineral oil/toluene (v/v) were used to prepare calibration standards. The calibration standards were prepared by dilution with $50: 50$ mineral oil/toluene (v/v) to give concentrations of $0,0.05,0.10,0.25,0.50,1.0,2.5$ and $5.0 \mu \mathrm{g} \mathrm{mL} \mathrm{m}^{-1}$ of TPPS. These provide concentrations at the lower limit of the quantification (LLOQ) and upper limit of quantification (ULOQ) for TPPS.

\section{Sample preparation and derivatisation procedure}

Mineral oil samples were stirred at room temperature, and then $5.0 \mathrm{~mL}$ of each sample was transferred to a round bottom flask to which $5.0 \mathrm{~mL}$ of toluene was added. For derivatisation, $20 \mathrm{mg}$ of TPP was added to each round bottom flask and the mixture was stirred at $60{ }^{\circ} \mathrm{C}$. Sufficient TPP is available for oil samples with a concentration of elemental sulfur up to $490 \mu \mathrm{g}$ $\mathrm{mL}^{-1}$. In this work, the samples were allowed to react for $3 \mathrm{~h}$ before being diluted 1:100 with 50:50 new mineral oil/ toluene(v/v) and loaded into autosampler vials. All samples were analysed by UHPSFC-MS immediately after preparation.

\section{UHPSFC method}

An Acquity UPC ${ }^{2}$ system (Waters Corp., Milford, MA, USA) was employed for all analyses. This system is equipped with a binary solvent manager, a heated column manager and an internal autosampler with a cooled sample tray. A HSS C18 SB column $(1.8 \mu \mathrm{m}, 3.0 \times 100 \mathrm{~mm})$ was used. The column was held at $40{ }^{\circ} \mathrm{C}$ in a column oven and $2.0 \mu \mathrm{L}$ of each sample solution was injected. A mobile phase modifier, methanol $(25 \mathrm{mM}$ ammonium acetate) was used. The supercritical (sc) $\mathrm{CO}_{2}$ back pressure of the system was set to 150 bar. A 3 min gradient elution was performed using the method described in Table 1 and a 1 min pre-run was used for column equilibration.

Before the analyses, three blank injections of methanol were performed to ensure system stability and cleanliness. Analyses commenced with triplicate injections of the calibration curve solutions (low concentration to high), each standard solution was followed by two blank injections. Study samples (triplicate measurements) were than analysed in a randomised order to minimise bias due to batch effects. The sequence of analysis is shown in Fig. 2.

\section{ESI-MS method}

A Waters TQD (Waters Corp., Milford, MA, USA) triple quadrupole mass spectrometer was used. An Internal Solvent Manager (ISM) was used to introduce make up solvent, metha-

Table 1 Inlet method - UHPSFC

\begin{tabular}{lll}
\hline $\begin{array}{l}\text { Time } \\
(\mathrm{min})\end{array}$ & $\begin{array}{l}\text { Solvent A } \\
\left(\mathrm{CO}_{2}\right) \%\end{array}$ & $\begin{array}{l}\text { Solvent B (methanol }+25 \mathrm{mM} \\
\text { ammonium acetate) } \%\end{array}$ \\
\hline 0.00 & 98 & 2 \\
2.00 & 60 & 40 \\
2.30 & 60 & 40 \\
3.00 & 98 & 2
\end{tabular}




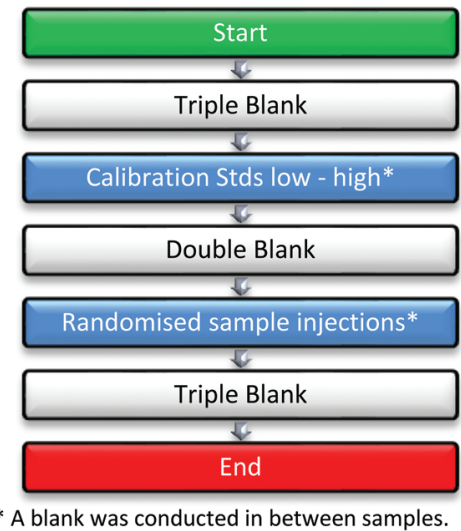

Fig. 2 Sequence of the samples during the analyses.

nol $+1 \%$ formic acid, after chromatographic separation at a flow rate of $0.5 \mathrm{~mL} \mathrm{~min}^{-1}$ to enhance ionisation and ensure a stable spray. The operating conditions for the positive ion ESI were as follows: capillary voltage $3.5 \mathrm{kV}$, cone voltage $20 \mathrm{~V}$, source temperature $150{ }^{\circ} \mathrm{C}$, desolvation temperature $500{ }^{\circ} \mathrm{C}$. Nitrogen was used as the desolvation gas at a flow of $300 \mathrm{~L} \mathrm{~h}^{-1}$. Full scan data over the $m / z$ range of $100-800$ were acquired for each injection. A positive ion ESI mass spectrometry single ion monitoring (SIM) method was created for the ion of interest $\mathrm{m} / \mathrm{z}$ 295, which corresponds to protonated TPPS [TPPS $+\mathrm{H}]^{+}$.

\section{Data processing}

Waters MassLynx ${ }^{\mathrm{TM}}$ software (version 4.1) (Waters Corp., Milford, MA, USA) was used to collect and process data for all the UHPSFC-MS experiments.

\section{Results and discussion}

\section{Method development}

TPP reacts quantitatively with elemental sulfur to give TPPS, ${ }^{22}$ which displays favourable ionisation properties under positive ion ESI conditions. Potential considerations associated with the use of TPP as derivatisation reagent are the possible occurrence of side reactions, such as oxidation to triphenylphosphine oxide (TPPO). However, probably due to the presence of the anti-oxidant (2,6-di-tert-butyl- $p$-cresol) in mineral oils, oxidation was not observed, and no modification of the derivatisation reaction conditions was required.

Different chromatography-MS approaches were investigated for quantification of TPPS in mineral oil. Electron ionisation (EI) provided an efficient method for detection of TPPS, however GC-MS proved to be unsuitable for reliable analysis of samples when mineral oil was present due to ion source contamination and subsequent rapid loss of sensitivity.

Therefore, atmospheric pressure ionisation techniques were explored in combination with SFC to provide reliable and short analysis times. The effectiveness of the ionisation of TPPS was explored using ESI for a $1 \mu \mathrm{g} \mathrm{mL} \mathrm{m}^{-1}$ standard solution, using a

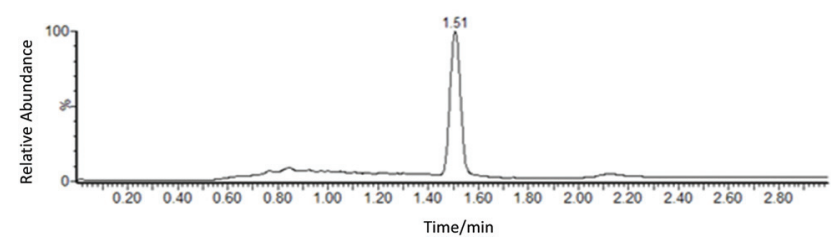

Fig. 3 ESI UHPSFC-MS SIM of TPPS at $1 \mu \mathrm{g} \mathrm{mL}^{-1}(\mathrm{~S} / \mathrm{N}=161)$.

Single Ion Monitoring (SIM) method ( $\mathrm{m} / \mathrm{z} 295)$. TPPS was found to undergo efficient ionisation, with reduced interference arising due to ionisation of the matrix. Under the selected conditions TPPS has a retention time of $1.5 \mathrm{~min}$, and the protonated molecule $\left([\mathrm{TPPS}+\mathrm{H}]^{+}\right)$is observed with a S/N of 161 (Fig. 3).

Positive ESI MS conditions for TPPS and the chromatographic method were optimised providing an analysis time of 3 min per sample. For quantification, an external standard calibration was employed where the ion at $m / z 295$ ([TPPS + $\mathrm{H}^{+}$) was used. Sample preparation was adapted to mitigate against the low solubility of TPPS in neat mineral oil, different solvents were studied based on their capacity to dissolve both TPPS and mineral oil. A 50:50 mineral oil/toluene mixture (v/v) provided acceptable solubility while maintaining good chromatographic efficiency and resolution.

\section{Method validation}

A range of analytical attributes were investigated, including linearity, lower and upper limits of quantification (LLOQ and ULOQ), reproducibility, carryover, matrix interference and derivative stability. Standard curves were found to be linear over the range measured $0-5 \mu \mathrm{g} \mathrm{mL}{ }^{-1}$, with correlation coefficients $\left(R^{2}\right)$ of 0.992 or better as determined over the three days used for validation. A representative calibration curve is shown in Fig. 4. A LLOQ of $0.05 \mu \mathrm{g} \mathrm{mL}^{-1}$ (S/N of 33) and an ULOQ of $5 \mu \mathrm{g} \mathrm{mL} \mathrm{m}^{-1}$ was used for all analyses. The concentration range covered by the standard curves equates to $0-10 \mu \mathrm{g} \mathrm{mL}{ }^{-1}$ of TPPS due to the 50:50 dilution of the samples prior to the derivatisation reaction. This concentration of TPPS corresponds to a range of $0-1.09 \mu \mathrm{g} \mathrm{mL}^{-1}$ of elemental sulfur. Carryover was assessed by calculating the concentration of TPPS present in the blanks performed in between standard analysis, and was only found present when analysing samples

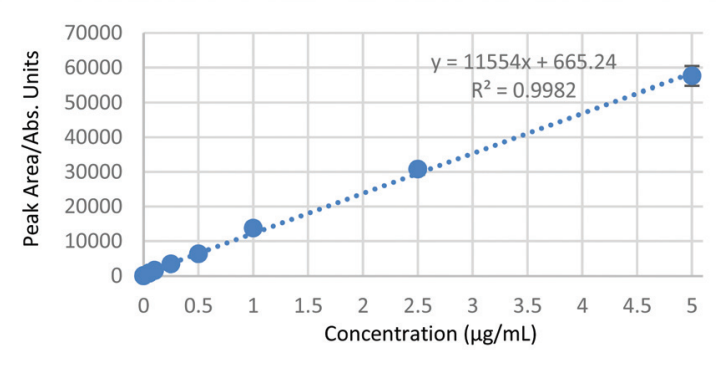

Fig. 4 Calibration curve for TPPS (number of replicate measurements = 4). 
with concentrations close to the ULOQ and presented a value of $\leq 0.5 \%$. TPPS was found to be stable for at least $24 \mathrm{~h}$ at ambient temperature or in the autosampler at $4{ }^{\circ} \mathrm{C}$. Intraday reproducibility was determined by the analysis of three standard samples with different concentrations over the range of 0-5 $\mu \mathrm{g} \mathrm{mL}^{-1}$ on three separate occasions, the method was found to be reproducible with a maximum relative standard deviation (RSD) of $2.03 \%$. In this work, a new calibration curve was prepared daily. Interference of the matrix on the concentration of TPPS is minimised as most components in mineral oil are not ionised by ESI, and a SIM method is employed. Furthermore, the retention time of TPPS is separated from the vast bulk of the oil components.

\section{Derivatisation selectivity and efficiency}

The selective reaction of TPP with elemental sulfur to form TPPS is the basis of this analytical method. Side reactions of the TPP with the other sulfur compounds present in the mineral oil would present false positives/increased concentrations, and are clearly undesirable. TPP reactivity with other representative sulfur compounds was studied using the conditions of the derivatisation method. It is not practical to test all sulfur compounds that are believed/known to be present in the mineral oil, so three representative sulfur compounds with different reactivities were studied. The compounds were dibenzyl disulfide (DBDS), benzothiophene (BT) and 4,6-dimethyldibenzothiophene (4,6-DMDBT). Each was reacted with TPP and analysed using the UHPSFC-MS SIM method. From the results obtained, it can be concluded that there are no products of the reaction between BT or 4,6-DMDBT with TPP. The reaction of the DBDS with TPP, presents only one trace product (TPPS) with an yield of $<1 \%$. This infers that the side reactions of the TPP with typical organosulfur compounds present in the oil are also likely to be negligible, giving confidence that TPPS formed during our studies will arise from the presence of elemental sulfur. These findings were later confirmed by GC-MS and ${ }^{31} \mathrm{P}$ NMR experiments. The progression/ conversion of the reaction was studied and determined by the reaction of a known concentration of elemental sulfur with TPP (excess, 1.1 eq.) using the conditions described in the derivatisation procedure. The concentration used was chosen in order to allow the reaction to be monitored by ${ }^{31} \mathrm{P} N \mathrm{NR}$ and by our UHPSFC-MS SIM method. After $3 \mathrm{~h}$, integration of the signal of TPPS in the ${ }^{31} \mathrm{P}$ NMR $\left(-4.58 \mathrm{ppm}, \mathrm{CDCl}_{3}, 162 \mathrm{MHz}\right)$ showed approximately $98 \%$ conversion of $\mathrm{S}_{8}$ to TPPS. The reaction was monitored for two more hours and this value did not change further. At the same time and during the same period, reaction progress was followed using our UHPSFC-MS SIM method, triplicate measurements were conducted after 1, 1.5, 2, 3.5 and $5 \mathrm{~h}$. The results are shown in Fig. 5, taking into consideration the results obtained from both experiments the derivatisation reaction time was set to $3 \mathrm{~h}$.

\section{Analysis of real mineral oil samples}

Following validation, the method was then applied to the analysis of real mineral oil samples from power transformers. To

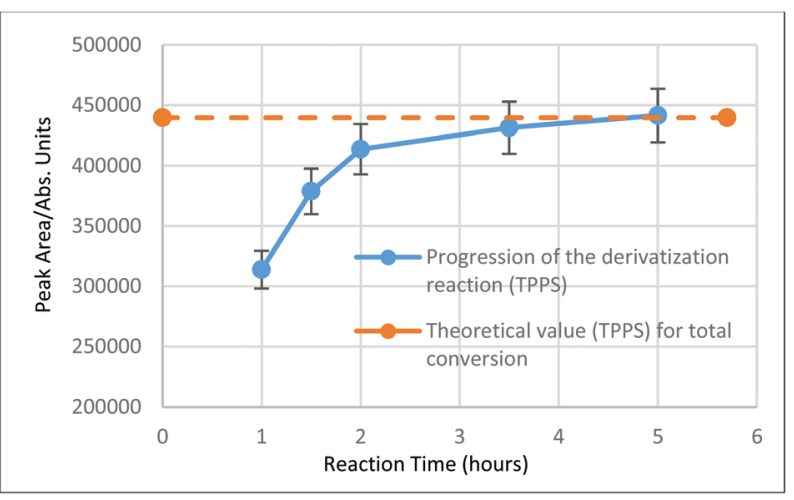

Fig. 5 Efficiency of the derivatisation reaction using the UHPSFC-MS.

Table 2 Reproducibility of the method when analysing real mineral oil samples

\begin{tabular}{lrll}
\hline${ }^{*}$ Values are in $\mu \mathrm{g} \mathrm{mL}{ }^{-1}$ of $\mathrm{S}_{8}$ & Sample A & Sample B \\
\hline Day 1 & Experiment 1 & 0.539 & 0.739 \\
& Experiment 2 & 0.542 & 0.737 \\
Day 2 & Experiment 3 & 0.524 & 0.694
\end{tabular}

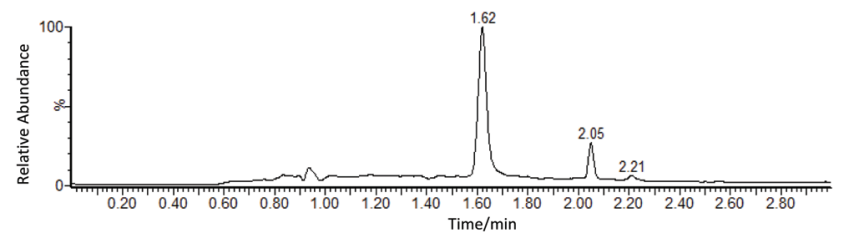

Fig. 6 SIM chromatogram obtained from a real mineral oil sample doped with elemental sulfur (TPPS peak at $1.62 \mathrm{~min}$ ).

test the reproducibility of this method when applied to real mineral oil samples, two different samples supplied by National Grid were analysed 3 times and on different days (Table 2). The example shown in Fig. 6, is the SIM chromatogram obtained from a real mineral sample oil that was doped with elemental sulfur prior to the derivatisation process. Inspection of the chromatogram shows a peak at the retention time of $1.62 \mathrm{~min},{ }^{24}$ corresponding to TPPS, and showing the protonated molecule $[\mathrm{TPPS}+\mathrm{H}]^{+}$in the mass spectrum. Two peaks at 2.06 and $2.21 \mathrm{~min}$, elute at retention times separated from TPPS and do not compromise accurate quantification. Furthermore, the different compositions inherent to each sample, due to different working conditions, did not have any negative effect on the analyses performed in this study.

\section{Conclusions}

The use of triphenylphosphine as a derivatisation reagent enabled high sensitivity and targeted analysis of elemental sulfur in mineral oil using an UHPSFC-MS method. The derivative, TPPS, enables sensitive, reproducible and specific 
analysis down to $0.05 \mu \mathrm{g} \mathrm{mL} \mathrm{m}^{-1}$ of TPPS. The use of ESI MS generates the protonated molecule, and results in low interference and background noise from the matrix. This provides a method capable to detect elemental sulfur in samples with varying composition and chemistry, including used/in service oils. This new UHPSFC-MS method has proven to be a fast, robust and reliable tool to identify oil samples that might present a risk for the power transformer.

\section{Conflicts of interest}

There are no conflicts to declare.

\section{Acknowledgements}

The authors acknowledge financial support from National Grid and EPSRC (EP/K039466/1).

\section{Notes and references}

1 M. Heathcote, J \& P Transformer Book, 2007, vol. 13, p. 40.

2 CIGRE Technical Brochure n³78, WG A2.32, 2009, 1, 5.

3 CIGRE Technical Brochure n625, WG A2.40, 2016, 1, 74.

4 IEC 60296, Fluids for Electrotechnical Applications: Unused Mineral Insulating Oils in Electrical Equipment, 2012, 4, 14.

5 IEC 60422, Supervision and Maintenance Guide for Mineral Insulating Oils in Electrical Equipment, 2013, 4, 11.

6 Standard DIN 51353, Detection of Corrosive Sulphur, 1985, $1,1$.
7 ASTM D1275-15, Standard Test Method for Corrosive Sulfur in Electrical Insulating Liquids, 2015, 4, 1.

8 ISO 5662, Petroleum products - Electrical Insulating Oils Detection of Corrosive Sulfur, 1997, 2, 1.

9 L. G. Borchardt and D. B. Easty, J. Chromatogr., 1984, 299, 471.

10 D. L. Struble, J. Chromatogr. Sci., 1972, 10, 57.

11 J. J. Richard, R. D. Vick and G. A. Junk, Environ. Sci. Technol., 1977, 11, 1084.

12 J. L. Guinon, J. Monzo, J. Garcia-Anton, C. Urena, J. Costa and J. Fresenius', Anal. Chem., 1990, 337, 372.

13 P. D. Clark and K. L. Lesage, J. Chromatogr. Sci., 1989, 27, 259.

14 H. Sid Kalal, A. A. Miran Beigi, M. Farazmand and Sh. A. Tash, Analyst, 2000, 125, 903.

15 B. R. Olofsson, Anal. Chim. Acta, 1985, 177, 167.

16 B. F. Taylor, T. A. Hood and L. A. Pope, J. Microbiol. Methods, 1989, 9, 221.

17 G. D. Woods and F. I. Fryer, Anal. Bioanal. Chem., 2007, 289, 753.

18 W. M. Geiger, S. McSeehy and M. J. Nash, J. Chromatogr. Sci., 2007, 45, 677.

19 B. Meyer, Chem. Rev., 1976, 76, 367.

20 J. Heilmann and K. G. Heumann, Anal. Chem., 2008, 80, 1952.

21 P. D. Bartlett and G. Meguerian, J. Am. Chem. Soc., 1956, 78, 3710 .

22 R. E. Pauls, J. Chromatogr. Sci., 2010, 48, 283.

23 ASTM D7800/D7800M-14, Standard Test Method for Determination of elemental sulfur in Natural Gas, 2014, 5,1 .

24 The shift of the retention time of the TPPS is due to small changes in the composition of solvent B. 